\title{
ROMEO AND JULIET Y “THE PROBLEM COMEDIES"
}

\author{
Susana Onega Jaén
}

"Shakespeare never wrote if it was possible to rewrite". Con esta categórica afirmación da comienzo Philip Hobsbaum en Tradition and Experiment in English Poetry ${ }^{1}$ al capítulo dedicado a las fuentes de Shakespeare, sintetizando la candente cuestión de las razones que pudo tener Shakespeare para utilizar todo tipo de fuentes en la confección de gran número de sus obras dramáticas. Y añade:

He had extraordinary powers of realising the implications latent in other people's plots. Indeed, he treated previous writings as raw material for his recreation. ${ }^{2}$

Desde este punto de vista, la originalidad de Shakespeare radica sobre todo en su capacidad para asimilar las más diversas influencias y para extraer de ellas elementos comunes que habrían pasado desapercibidos a otros observadores más superficiales, de manera que, en palabras de Hobsbaum, la obra de Shakespeare "is the supreme example in English of the union of tradition with experiment"3.

Harding Craig por su parte, en "Motivation in Shakespeare's Choice of Materials" 4 , justifica la utilización que Shakespeare hace de sus fuentes en función ce la filosofía renacentista del arte como algo capaz de recrear los diversos avatares de la vida del hombre con la fidelidad de un espejo, ael cual puecie derivarse una lección, un "exemplum". Según esto, lo importante no sería tanto la

1. Tradition and Experiment in English Poetry. Philip Hobsbaum. The Macmillan Press Ltd. London 1979, p. 89.

2. Tradition and Experiment... Op. cit. p. 89.

3. Tradition and Experiment... Op. cit. p. 89.

4. 'Motivation in Shakespeare's Choice of Materials". Hardig Craig. En: Shakespeare's Survey. vol. 4. 1951-68, pp. 26 a 34, p. 27. 


\section{SUSANA ONEGA JAEN}

originalidad o no de una obra concreta, sino su capacidad de producción del efecto que se busca en el público, ya que, básicamente, las situaciones que dichas obras de arte reflejan son siempre las mismas, pues se refieren siempre al hombre considerado como microcosmos. Consecuentemente, lo que habría que establecer a la hora de juzgar la validez o no de cada una de sus piezas teatrales es si al tomar una obra ajena como base de su propia versión, Shakespeare se estaba dejando llevar por la tentación de hacer suyo un éxito asegurado, o si por el contrario la utilización de su fuente argumental tiene que ver con un esquema mental del autor con respecto a la totalidad de su obra, es decir, si es posible considerar cada pieza teatral de Shakespeare como un eslabón coherente e inelusdible del conjunto.

Leo Salingar ${ }^{5}$ se expresa en términos parecidos cuando admite lo fácil que resultaría caer en el error de creer que Shakespeare necesitaba inspirarse en una obra anterior cada vez que tenía que escribir algo, peligro que, en su opinión, se acrecienta cuando se trata de obras tan populares como Romeo and Juliet, ya que, antes que él, cuando Shakespeare tenía unos 20 años, una docena por lo menos de autores isabelinos se habían referido a Romeo como a un amante famoso y desgraciado, o habian condenado su matrimonio secreto con Julieta. Cuando Shakespeare se decide a escribir su propia versión de la historia, el tema de los amantes de Verona es enormemente popular en la Italia renacentista, y se repiten versiones más o menos adulteradas en Francia e incluso en España, en obras como Calixto y Melibea o Los amantes de Teruel. Ante tan abrumadora evidencia es lícito preguntarse si Shakespeare pretendió únicamente tomar su parte de popularidad, aportando su propia versión de la obra o si, por el contrario, es posible justificar la existencia de Romeo and Juliet como un hito necesario en el conjunto dramático de Shakespeare. Esta pregunta será el punto de partida de nuestro estudio. Para responderla será necesario constatar el lugar que Romeo and Juliet ocupa sobre todo entre otras obras que como ésta proceden de un tipo común de fuente: la "novella" italiana. Para este propósito nos resultará de gran utilidad el libro de Leo Salinger: Shakespeare and the Traditions of Comedy ${ }^{6}$, en el que se sintetiza la evolución general de la producción cómica de Shakespeare, y concretamente de las "novella comedies".

Shakespeare escribió Romeo and Juliet en 1595, es decir, en un momento en que confluían en el escritor diversas influencias literarias importantes. De un lado, la tradición medieval que, desde los tiempos de Chaucer hasta 1570 , comprendía las formas dramáticas dominantes en Inglaterra (lo mismo que en el resto de Europa): piezas religiosas, como martirios o vidas de santos; morales, como misterios o ciclos de la Biblia; y seculares, como los romances y las "far-

5. Shakespeare and the Traditions of Comedy. Leo Salingar. Cambridge University Press. Cambridge 1974-1976.

6. Shakespeare and the Traditions... Op. cit. 
sas", obras éstas que Shakespeare ha visto representar desde niño durante ei Carnaval, es decir, en el "time of misrule", que podía abarcar desde Epifanía hasta la Cuaresma. Los personajes de este teatro popular solían ser caballeros, juglares, damiselas desgraciadas pero inocentes que vagan sin rumbo fijo, padres venerables desterrados injustamente, y duques o príncipes desposeídos. En los romances morales aparecía también frecuentemente un juez al que se pide que haga justicia por encima incluso de consideraciones personales, a fin de que se destierre el vicio y triunfe la virtud. Este personaje aparecerá de forma más o menos encubierta en numerosas obras de Shakespeare. En cuanto al mundo en que se mueven estos personajes de romance es siempre un mundo ideal, de ensueño, desligado de limit aciones espacio-temporales, donde los encantamientos son posibles y los disfraces y las triunfales escenas de "reconocimiento" en las que la Fortuna juega un papel decisivo, conducen siempre a una resolución positiva.

Por otra parte, en los primeros años de la vida de William Shakespeare, comienzan a florecer en Inglaterra las primeras compañías de actores profesionales. El primer teatro estable de Londres data de 1576 , lo que indica un progresivo aumento del interés popular por las representaciones teatrales, reflejado a su vez en la creciente demanda de nuevas obras que representar que se hace sentir con fuerza en Inglaterra en torno a esta fecha. En círculos intelectuales, Oxford y Cambridge sobre todo, comienzan a desempolvarse antiguos manuscritos latinos que serán adaptados al inglés por autores como Gascoigne y después Lyly o Peele, difundiéndose seguidamente esta tendencia entre autores que ya escriben para los teatros públicos como Marlowe, Kyd, Greene y el propio Shakespeare ${ }^{7}$.

Entre 1567-1582, por tanto, las nuevas obras teatrales que se escriben en Inglaterra son de dos tipos contrapuestos: unas, de corte neoclásico por su forma y derivaciones, son representadas ante auditorios educados en la tradición humanista; y otras, como los interludios morales o los romances de tradición medieval, que se destinan a todos los públicos.

Coincidiendo con el auge de la profesión teatral en torno a 1576, las autoridades eclesiásticas inglesas, temiendo que aquella se convierta en una seria amenaza para las buenas costumbres de los ingleses, inician un ataque generalizado de tipo administrativo y religioso a las comedias seculares, al que seguirá a su vez otro más decisivo de tipo cultural y literario que rechaza cualquier atisbo de reminiscencia medieval en favor de una renovación que (a imitación del renacimiento italiano) se basa en textos de origen clásico, griego y latino. Ahora se estudian con entusiasmo las comedias de Plauto y Terencio y se toman como objetivos primordiales de calidad en una comedia los presupuestos estipulados por Horaeio en su Ars Poetica. Frente a la falta de verosimilitud y coherencia

7. A este respecto, vid: The Drama to 1642: Parts One and Two. En: The Cambridge History of English Literature, vols. V y VI. 
de obras medievales que hacen discurrir la acción en diversos paises simultáneamente o la dilatan para cubrir varios años, se ofrece ahora como ideal a alcanzar la unidad de espacio, tiempo y acción, la supresión de prolegómenos poco interesantes y el inicio de la obra "in media res", en el centro de interés, volviendo atrás si es necesario para dar algún dato sustancial, o encomendando dicha tarea al Prólogo.

La trama central de una comedia nueva, es decir, procedente de autores que, como Plauto y Terencio, se basaban en Menandro, consiste, en opinión de Northrop Frye ${ }^{8}$, en el desarrollo del mito de Edipo, es decir trata de los esfuerzos de un hombre joven para engañar a su oponente, generalmente viejo, y poseer a la muchacha que ha elegido para sí. El oponente suele ser el propio padre del joven y la madre actúa como aliada del hijo en la lucha por la posesión de la mujer, que suele ser una esclava o concubina, y por tanto no puede aspirar a casarse. La resolución de este conflicto básico descansa en una escena de "cognitio", esto es, de reconocimiento del alto origen de la joven, lo que hace posible el matrimonio. En la comedia griega y latina, lo normal es que el joven venza al anciano, no al revés. Northrop Frye hace ver asimismo ${ }^{9}$ la existencia de un lado social en la trama de la Nueva Comedia, que se manifiesta en la atmósfera general de reconciliación que hace posible el matrimonio en el último acto. Cuando el héroe consigue superar todas las resistencias y dificultaảes y hacerse con la heroína, los personajes positivos se ponen de su parte y dan lugar al establecimiento de un nuevo orden social, que oristaliza en el último acto, cuando se reúnen todos los personajes en el escenario para celebrar el banquete nupcial. Los personajes que obstaculizaban el nacimiento de esta sociedad libre son siempre "humours": el avaro, el neurótico, el hipócrita, el pedante, etc., es decir, personajes ridículos por su sujeción a una forma de comportamiento impuesta por su falta de auto-conocimiento, de comprensión de sí mismos.

En lo que respecta a Shakespeare, si el romance medieval, con sus viajes $\mathrm{y}$ sus peregrinaciones del mundo del orden al espacio verde, sus maravillosas concatenaciones de casualidades y su aceptación del cambio de fortuna como consecuencia ineludible del ciclo vital del hombre, sirve para ilustrar el punto de partida de su dramática, su utilización de los presupuestos de la comedia clásica nos muestra la dirección en que irá moviéndose su mente. Este cambio de orientación de su interés, irá acompañado, como demuestra Leo Salingar ${ }^{10}$, por un giro en su criterio selector en la elección de fuentes literarias.

Hasta Measure for Measure Salingar clasifica como derivadas de fuentes clásicas o eruditas italianas 4 comedias: The Comedy of Errors, The Taming of the Shrew, The Merry Wives of Windsor y Twelfth Night. Todas ellas incluyen

8. "The Argument of Comedy". Northrop Frye. En: Shakespeare: Modern Essays in Criticism. Oxford University Press. London-New York, 1957-1967.pp. 79-80.

9. "The Argument of Comedy". Op. cit. p. 81.

10. Shakespeare and the Traditions... Op. cit. p. 298. 
transformaciones de la identidad, tal como ocurre en las comedias del bosque, pero en las de origen clásico estas transformaciones se producen solamente con engaños debidos a la suerte y en general su trama se desenvuelve en la ciudad o en la corte, no en el bosque. No hay episodios de magia $y$, lo mismo que sus modelos clásicos, vienen a representar la capacidad de los ciudadanos corrientes, ayudados en todo caso por la Fortuna, para resolver sus asuntos particulares sin necesidad de huir al bosque para sustraerse a la autoridad dictatorial del Príncipe.

En As You Like It, por el contrario, los amantes y otros personajes principales tienen que huir al bosque para escapar al poder del Príncipe y las dificultades que se originan en la Corte se resuelven favorablemente. Los amantes se unen, el legítimo duque encuentra a su hija y es llamado a ocupar su trono sin necesidad de tener que luchar por él, porque el usurpador ha sufrido una conversión a manos de un anciano religioso al acercarse al lindero del bosque. Una reconciliación y huida similares del poder al bosque, de la ciudad al "green world", tiene lugar en Two Gentlemen of Verona y en A Midsummer Night's Dream, por lo que Leo Salingar las agrupa como comedias procedentes de romances pastorales o de los romances escenificados de finales de la Edad Media, siendo posible agrupar con ellas a Love's Labour's Lost porque la acción tiene lugar en un parque y también en el bosque y porque el rey y sus amigos están alli para evadirse de sus responsabilidades y es en este espacio verde donde los caballeros encuentran a las damas francesas y se enamoran, liberándose de sus prejuicios contra las mujeres. Obras como éstas son posiblemente las primeras que Shakespeare tuvo oportunidad de ver, y volverá a utilizar esta serie de ideas de la literatura pastoral renacentista y de los romances medievales para sus últimas comedias, desde Pericles en adelante.

Pero existen otras 4 comedias que no pueden clasificarse ni como comedias del nundo verde ni como clásicas o eruditas: The Merchant of Venice, Much Ado About Nothing, All's Well that Ends Well y Measure for Measure. Todas ellas se escribieron después de $A$ Midsummer Night's Dream y también después de Romeo and Juliet, entre 1596 y 1604, es decir, hacia la mitad de la carrera dramática de Shakespeare, coincidiendo por una parte con la serie de Henry IV y con Henry $V$ y por otra con el inicio del interés de nuestro autor por la tragedia. Las tramas principales de estas 4 obras vienen directa o indirectamente de un tipo común de fuente literaria, la "novella" italiana: The Merchant of Venice de Ser Giovanni Fiorentino; la trama de Hero y Claudio en Much Ado About Nothing de Bandello (lo mismo que Romeo and Juliet), All's Well That Ends Well de Boccaccio (como gran parte de la trama principal de Cymbeline más tarde) y Measure for Measure de Giraldi (como Othello, la siguiente obra de Shakespeare), y por esta razón Salinger las agrupa bajo el denominador común de "novella comedies". El tono de estas comedias es por regla general mucho

11. A este respecto ver el interesante artículo de A. P. Rossiter: "The Problem Plays". En: Shakespeare: Modern Essays in Criticism. Op. cit. pp. 263 a 282. 
más serio que el de las demás comedias de Shakespeare, a veces incluso tenso y sombrío hasta el punto de rozar con la tragedia, y por ello es lícito denominarlas también, siguiendo a F. S. Boas" , "problem comedies". Estas 4 comedias incluyen escenas de disfraz o de engaño y también la Fortuna, o la casualidad, viene a ayudar a la heroína, pero tanto ella como sus partidarios tienen ahora que enfrentarse con un enemigo deliberado: Shylock, Don John, Bertram o Angelo y, pese a todos sus esfuerzos, la acción de los oponentes no puede ser anulada sólo con la suerte, sino que es necesario recurrir a la ley. En este sentido se acercan al tema medieval del juez que debe hacer justicia y también al tema del mundo verde en cuanto que implican una consolidación de la suprema autoridæd del Príncipe, pero el enfoque que se da al tema es mucho más problemático que romántico.

Por otra parte, mientras que en las demás comedias de Shakespeare la trama conduce a la celebración de una boda al final de la obra, en las 4 comedias problemáticas ocurre algo diferente, ya que la boda se legaliza o la unión se consuma al principio o a la mitad de la obra, no al final, y después este matrimonio o esta unión se interrumpe bruscamente y nunca llega a completarse. Entonces la heroína o sus partidarios deben llevar a cabo una tarea difícil o resolver una paradoja legal antes de poder llegarse a un ambiente de celebración en el escenario. Nada más celebrarse la ceremonia nupcial en The Merchant of Venice, indica Salingar ${ }^{12}$, Portia despacha a Bassanio de Belmont para que salve a Antonio y luego ella misma le sigue secretamente. En Much Ado About Nothing Benedick se queja en la iglesia diciendo "This looks not like a nuptial". En All's Well That Ends Well Bertram se casa con Helena bajo amenazas, pero se niega a compartir su lecho; y por unirse secretamente, Claudio es condenado a muerte en Measure for Measure. Está claro que todas ellas son historias de bodas interrumpidas y que, tal como postula Salingar, cuando Shakespeare se decidió a tomar estas historias como base a sus comedias lo hizo porque buscaba conscientemente desvelar las posibilidades dramáticas de esta trama. En este sentido es significativo que en las tramas principales de otras 3 obras derivadas de una "novella", Romeo and Juliet, Othello y Cymbeline, la atención se centre asimismo en una boda interrumpida. Evidentemente Shakespeare utiliza las fuentes italianas de "novella" para un tipo especial de trama, concretamente una que combina dos elementos: las bodas interrumpidas y la crisis en torno a la justicia, y utiliza este material conjuntamente para comedias y tragedias.

Desde este punto de vista, $A$ Midsummer Night 3 Dream, escrita al mismo tiempo que Romeo and Juliet, puede considerarse como una alternativa cómica a la historia de amor de Romeo and Juliet, mientras que las comedias problemáticas, con sus historias de bodas interrumpidas y felizmente reanudadas representan una solución intermedia. Es decir, todas estas obras son experimentos sucesivos con los que Shakespeare intenta responder a lo que era básicamente una presión emocional continua.

12. Shakespeare and the Traditions... Op. cit.pp. 302 a 303. 
Shakespeare no llega a resolver este conflicto entre ley y amor completamente pero su tratamiento dramático varía y el énfasis cambia de orientación tras escribir Romeo and Juliet: Después de asistir a una representación de esta obra el espectador moderno se siente conmovido generalmente por dos sentimientos contrapuestos, de un lado está la exhuberancia poética del lenguaje shakespeariano en esta obra; de otra, la mala suerte de los trágicos amantes. Para un espectador de nuestros días, este segundo hecho, la concatenación de casualidades (de hechos incontrovertibles y absurdos, que diría William Faulkner) tan injustificados a veces como la detención de Fray Juan en su camino hacia Mantua para avisar a Romeo, conllevan un cierto desencanto, pues obligan al espectador a un enorme esfuerzo de aceptación con que suplir una falta de verosimilitud, desproporcionada según nuestros cánones modernos de realismo. Este defecto de la obra, si puede llamarse así, resta patetismo a la tragedia como tal, siendo contrapesado únicamente por la fuerza extraordinaria del lirismo del lenguaje shakespeariano.

La trama de Romeo and Juliet, supeditada a diversos golpes de mala suerte, se separa del resto de las tragedias escritas por Shakespeare que han servido para acuñar la expresión "shakespearian tragedies" reclamando un puesto al lado de las comedias. Para expresar esta diferencia de tono, los estudiosos de Shakespeare han utilizado a veces la expresión "tragedia romántica" para definirla. Romántica en el sentido medieval de obra que alberga dentro de sí categorías de irrealidad, de supresión de las leyes de la lógica y que confiere a la Rueda de la Fortuna un papel decisivo en los éxitos y fracasos que constituyen la vida del hombre.

Sin embargo, la importancia de la mala suerte en el desarrollo de la tragedia no agota las posibilidades de interpretación de la obra. Llevando a la práctica los postulados teóricos de la Nueva Comedia, Shakespeare ha construido una tragedia que comienza "in media res", es decir, con el momento culminante en que los dos "star-crossed lovers" se encuentran y se enamoran y, siguiendo también las normas establecidas por Horacio, ha relegado al Prólogo la tarea de situarnos en escena; éste será, por tanto, quien nos dé una clave inapreciable para la comprensión del todo:

Two households, both alike in dignity, In fair Verona, where we lay our scene,

From ancient grudge break to new mutiny, Where civil blood makes civil hands unclean.

$$
\text { (I - i - 14) }
$$

Sorprendentemente, el Prólogo no nos habla de amor, ni de Romeo y Julieta, sino de dos familias de igual status social, enfrentadas entre sí por una "antigua querella". Se trata por tanto de una situación típicamente cómica en el sentido de que el panorama que se nos ofrece al levantarse el telón es el 
de una sociedad que vive en un estado de guerra civil, es decir, de injusticia social. Como en el esquema tipificado por Northrop Frye como prototipo de la Nueva Comedia, Romeo and Juliet dramatiza la lucha de un joven enamorado que pugna por arrancar a la joven que ha elegido para esposa de las garras de un "senex", si bien, a diferencia de aquella, el oponente a sus deseos es el propio padre de Julieta, quien, en vez de pretender la posesión de la joven para sí mismo, la quiere para entregársela a Paris, que es joven y arrogante como Romeo, digno esposo, sin duda, de Julieta, de no haber caído ésta en las redes de un amor a primera vista. Con la introducción de Paris en el esquema, Shakespeare racionaliza en gran medida el planteamiento clásico, dándole mayor verosimilitud $y$ añadiendo patetismo al conflicto en cuanto que no se trata ya de luchar contra un viejo libidinoso, un "humour" risible y despreciable, sino contra un joven noble que tiene tanto derecho a la mano de Julieta como pueda tener Romeo y que cuenta además con el beneplácito de los Capuleto. Pero las razones de los padres de Julieta están viciadas por un prejuicio de base: su negativa a consentir la entrada en su familia de Romeo se debe únicamente a aquella antigua querella entre Capuletos y Montescos; por ello es injusto y no debe ser obedecido. Al casarse secretamente, Romeo y Julieta están intentando forzar la reconciliación de sus respectivas familias, la supresión del estado de injusticia que se estableció a causa de aquel "ancient grudge" y precisamente ésta será su victoria, aunque tengan qu lograrla al precio de su muerte. Vista así, la mala suerte de Romeo y Julieta no es ya el producto de una fortuna ciega o insensible, sino el resultado inexorable de la justicia divina, que impone un castigo antes de perdonar la ofensa, el antiguo error. Al igual que en las comedias griegas, el mundo de sometimiento conduce a una infracción de la ley, a una ruptura del orden por un miembro joven e inconformista de la comunidad, y de este acto de afirmación de la propia libertad surgirá un nuevo estado de orden, y de paz.

En las comedias del mundo verde la realización del deseo se asocia con un estado de irrealidad, de suspensión de leyes naturales y de festividad, y el mundo del amor se identifica con el mundo de lo moralmente deseable. En las comedias problemáticas, por el contrario, el amor vence sólo tras una lucha enconada entre ley y clemencia,entre justicia y piedad que refleja una obsesión personal de nuestro autor. En Romeo and Juliet para reparar el daño, para olvidar el rencor, es necesario morir - en la Verona renacentista no hay un Dios clemente que detenga la mano que va a sacrificar a Isaac-; Romeo y Julieta mueren, no sólo porque han nacido con mala estrella, sino porque son inocentes y deben sacrificarse para redimir a los demás. Contemplado a esta luz, el final del último acto, cuando aún calientes los cuerpos sin vida de los trágicos amantes, cae el telón sobre el fondo del escenario para resaltar la entrada en el primer plano del Príncipe de Verona y de los miembros supervivientes de ambas familias, adquiere una importancia vital que no ha sido comprendida por algunos adaptadores modernos de la obra, que la suprimen por encontrarla superflua. En efecto, al ocultar al público la triste visión de los cuerpos y concentrar su atención en el primer plano del escenario, Shakespeare busca subrayar una escena típicamente 
cómica de reconciliación general: como árbitro absoluto, detentador del poder y de la ley, el Príncipe-magistrado reúne a Capuletos y Montescos en torno suyo y, coreado por la totalidad de los personajes que han ido tomando parte en el desarrollo de la obra, exige el cese de las hostilidades y el perdón mutuo, haciendo posible así el nacimiento de una nueva sociedad más justa y también más libre. El sacrificio de las vidas inocentes de Romeo y Julieta ha conducido a esta escena de "vita nuova", de reconciliación, y el telón cae sobre una comunidad redimida por su mística comunión con su sangre y con su carne. Con esta escena, Shakespeare afirma su concepción cristiana del mundo y concluye el círculo perfecto que constituye la obra, traspasando el estadio individual de la tragedia para conducirnos al plano social de la comedia, confirmando al propio tiempo la validez del postulado teórico de Northrop Frye según el cual la tragedia no es más que una comedia interrumpida antes de llegar al final. De esta manera, sin apenas apercibirnos, Shakespeare nos hace traspasar las barreras teóricas entre los géneros y como una de sus queridas mascaradas en el desfile de Carnestolendas, nos encoge el corazón con una lágrima sangrienta en el ojo derecho, mientras nos guiña, pícaro, el otro ojo y nos sonríe con la mitad de la cara.

$\mathrm{Al}$ compartir una trama trágica con una estructura de comedia, Romeo and Juliet se sitúa en un lugar singular dentro de la obra dramática de Shakespeare, ocupando una posición intermedia entre las comedias problemáticas que preludian ya un oscurecimiento general del panorama, y las tragedias mayores, con las que culminará dicha tendencia. De este modo, la producción de Romeo and Juliet se justifica plenamente. 
